\title{
Concept mapping of knowledge structures to visualize the nature of expertise in clinical education.
}

\author{
Ian M. Kinchin \\ King's College London \\ ian.kinchin@kcl.ac.uk
}

\begin{abstract}
Motivation - To provide a model of university teaching that finds a middle way between the medieval transmission of knowledge and the current focus on student learning needs. Research approach - A synthesis of various studies is presented that have employed concept mapping and the direct observation of teaching. Findings/Design - The model presented provides an approach that can support student learning in a manner that is practicable for university teachers whilst helping to address the tensions between teaching and research. Research limitations/Implications - The findings are based upon detailed analysis of two components of meaningful learning (knowledge and action) without paying similar attention to the affective domain. Originality/Value - The research makes a contribution to the practical needs of university teachers in creating an evidence-informed pedagogy. Take away message Visualization of learning and teaching can help to develop a more authentic pedagogy for higher education, based on the notion of expertise.
\end{abstract}

\section{Keywords}

Concept maps, visualization, pedagogy, university teaching.

\section{INTRODUCTION}

Concept mapping developed by Novak (1998), is a graphical tool used to represent links between ideas that has been used effectively in a variety of contexts to enhance learning. A concept map is an attempt to make explicit internally held mental models that usually remain private so that they can be reviewed with others. Organization of knowledge can facilitate learning by making the material to be learned more predictable and so reducing the learning effort required. Concept maps are increasingly used in teaching health professionals to summarize learning, promote critical thinking skills and meaningful learning or as an assessment strategy. The application of concept mapping to the qualitative analysis of knowledge structures has allowed the visualization of the process of learning at university in novel ways that emphasize the organization of understanding.

The revealed transformation of knowledge structures within the teaching and learning process has led to a description of clinical expertise that provides functional links between expert knowledge and clinical practice. The usual reference to these links as 'tacit' or 'intuitive' has previously helped to complicate the issue of how to develop a clinical pedagogy that helps students to develop appropriate links between theory and practice and a trajectory towards expertise. Visualization allows the surfacing and subsequent focus on key attributes, and is seen as particularly important in the development of clinical reasoning.

\section{VISUALIZING EXPERTISE}

A reconceptualisation of expertise as a dynamic transformation of knowledge structures relating competence and comprehension can be represented by chains of practice and networks of understanding revealed by concept mapping. This view of expertise has provided us with a threshold concept for the evolution of university pedagogy, whilst moving away from the problematic binary of student/teacher centredness. It also rejects reductionist notions of expertise fostered by an audit culture, preferring an integration of the elements of professional practice. This model facilitates the academic reclamation of pedagogy, placing subject specialists at the centre of pedagogic developments and provides a mechanism to initiate and monitor a more transactional curriculum. The principle characteristics of experts in any domain is that they posses an extensive and highly integrated body of knowledge related to their discipline. This is coupled with the ability to perceive patterns in large amounts of information and to process their responses quickly and efficiently. Uncovering the knowledge bases held by experts to gain insight of the nature of the structures that might be 
indicative of expert understanding has led to the use of concept mapping as an exploratory cognitive task analysis tool (Hoffman and Lintern, 2006).

The ability to visualize reasoning processes is considered to represent one of the first steps in the formation of the cognitive skills that are necessary for professional practice. Visualization of knowledge structures through concept mapping has enabled us to separate the chains of practice that are manifest in teachers' actions from the underlying networks of understanding. Chains are indicative of procedural sequences that characterize observable practice and have been described as indicators of goal-orientation. This seems entirely appropriate in clinical practice, where the goal of clinical competence is the effective treatment of patients. However, if there are no links with an underlying understanding, the chain may be seen as blindly following a recipe. Networks indicate understanding that is integrated and wholistic. So, for example, knowing there are several alternative treatments with varying consequences is not the same as being able to select the most appropriate one within a clinical context. If this was the case, academic study would not need to be backed by clinical training.

\section{CLINICAL TEACHING}

In the clinical teaching arena, an effective teacher needs to be able to articulate knowledge that would normally be tacit for a practitioner not engaged in instruction. It is precisely the articulation of this tacit knowledge that is facilitated by the concept mapping tool. The tacit knowledge that needs to be placed in the public arena for teaching is found connecting the chains of practice that are manifest in the teacher's actions and the underlying network of understanding that is usually held privately (Kinchin, Cabot and Hay, 2008).

The student needs to gain experience in converting between complementary chains and networks that can be modeled for the student, once the teacher has recognized them. Engagement in concept mapping activities allows the teacher to recognize the existence of the structures and allows him/her to make them public to the students. Concept mapping also slows down the process (that is usually automated) to facilitate its examination. So, for example, the typical structure of a clinical procedure would be a chain of practice that would be communicated to the student. The student's competence would be assessed through his/her ability to reproduce that chain under varying conditions and with various patients. The student's developing expertise, however, must be assessed through his/her ability to relate the chain of practice to the underlying network of understanding, and explaining how the elements are linked, and how and why the chain of practice should be modified in response to changes of context. This represents a shift in the emphasis of the transactions between teachers and students from fixed end-points to linking activities.

\section{REFERENCES}

Hoffman, R.R. \& Lintern, G. (2006) Eliciting and representing the knowledge of experts. In: Ericsson, K.A., Charness, N., Feltovich, P.J. and Hoffman, R.R. (Eds.) The Cambridge handbook of expertise and expert performance. Cambridge University Press. pp. $203-222$.

Kinchin, I.M., Cabot, L.B. and Hay, D.B. (2008) Visualizing expertise: towards an authentic pedagogy for higher education. Teaching in Higher Education, 13, 3, 315 - 326.

Novak JD (1998) Learning, Creating, and Using Knowledge: Concept maps ${ }^{\mathrm{TM}}$ as facilitative tools in schools and corporations. Mahwah, NJ. Lawrence Erlbaum Associates. 